\title{
One Technique to Enhance the Resolution of Discrete Fourier Transform
}

\author{
Ivan Kanatov ${ }^{1}$, Dmitry Kaplun ${ }^{1}{ }^{(0)}$, Denis Butusov ${ }^{2}{ }^{\circledR}$, Viacheslav Gulvanskii ${ }^{1, *}$ and \\ Aleksander Sinitca ${ }^{1}$ \\ 1 Department of Automation and Control Processes, St. Petersburg Electrotechnical University "LETI", \\ Saint Petersburg 197376, Russia; ivankanatov@yandex.ru (I.K.); dikaplun@etu.ru (D.K.); \\ amsinitca@etu.ru (A.S.) \\ 2 Youth Research Institute, St. Petersburg Electrotechnical University “LETI”, Saint Petersburg 197376, Russia; \\ dnbutusov@etu.ru \\ * Correspondence: vvgulvanskii@etu.ru; Tel.: +7-965-777-7063
}

Received: 31 December 2018; Accepted: 14 March 2019; Published: 18 March 2019

\begin{abstract}
Discrete Fourier transform (DFT) is a common analysis tool in digital signal processing. This transform is well studied and its shortcomings are known as well. Various window functions (e.g., Hanning, Blackman, Kaiser) are often used to reduce sidelobes and to spread the spectrum. In this paper, we introduce a transformation that allows removing the sidelobes of the Fourier transform and increasing the resolution of the DFT without changing the time sample. The proposed method is based on signal phase analysis. We give the comparison of the proposed approach with known methods based on window functions. The advantages and disadvantages of the proposed technique are explicitly shown. We also give a set of examples illustrating the application of our technique in some practical applications, including engine vibration analysis and a short-range radar system.
\end{abstract}

Keywords: Fourier transform; discrete Fourier transform; fast Fourier transform; sidelobes reduction; DFT resolution

\section{Introduction}

It is often necessary to estimate the frequency of a mono-harmonic signal in spectral analysis or to detect the center frequency of a narrowband signal. When we apply discrete Fourier transform (DFT), giving the output array size as a power of two, the frequency of the analyzed signal rarely coincides with the center of one of the DFT samples, whose frequency is exactly known [1].

$$
X_{k}=\sum_{n=0}^{N-1} x_{n} e^{-\frac{2 \pi i}{N} k n}, \quad k=0, \ldots, N-1
$$

where $N$-the number of signal values measured over a period, as well as the number of decomposition components; $x_{n}, n=0, \ldots, N-1$-measured signal values (at discrete time points with numbers $n=$ $0, \ldots, N-1)$; and $X_{k}, k=0, \ldots, N-1$ complex amplitudes of sinusoidal signals, composing an initial signal. Since the amplitudes are complex, it is possible to calculate both the amplitude and phase at the same time, $k$ is the frequency index. The frequency of the $k$-th signal is equal to $k / T$, where $T$ is the period of time during which the input data is taken.

In this case, due to the spectral leakage, the mono-harmonic signal spectrum obtained by discrete Fourier transform (DFT) looks like it is shown in Figure 1.

One can see that the maximum of the spectrum lies between the centers of samples with indices 11 and 12. The actual mono-harmonic signal has a frequency of $10.5 \mathrm{~Hz}$. In this case, the error of the frequency measurement is equal to the half of the distance between the adjacent samples. It can be 
calculated that there are two mono-harmonic signals with frequencies of 10 and $11 \mathrm{~Hz}$ in the spectrum, but in this example it is not possible to definitely determine the frequencies. The increase of the frequency resolution can be achieved by increasing the resolution of the DFT, but this would increase the computational complexity as well.

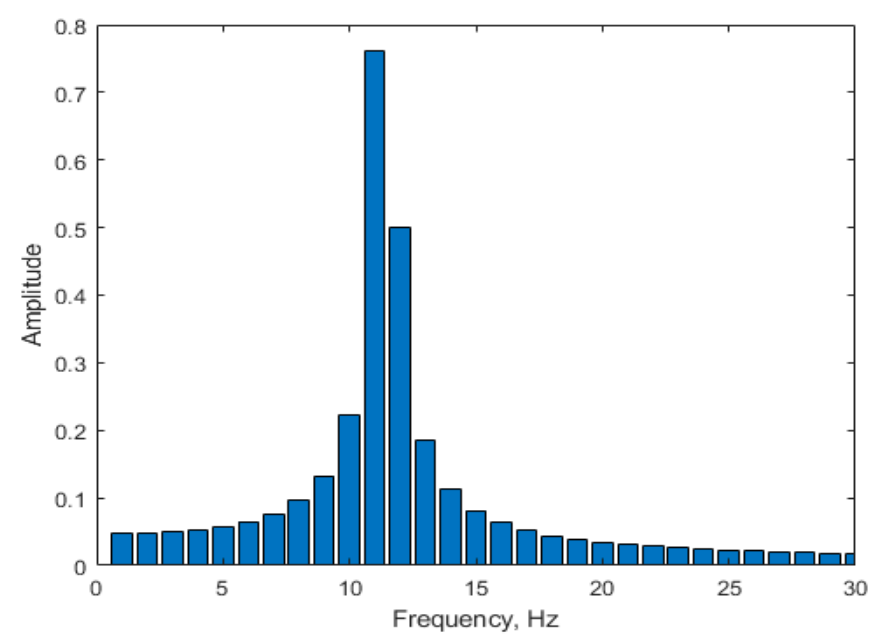

Figure 1. Illustration of the spreading spectrum of the DFT.

The procedure of signal spectral transformation for the DFT algorithm can be described as the procedure of dividing the signal among the frequencies of a set of bandpass filters. In the ideal case, these bandpass filters should have rectangular magnitude response with minimal overlap of adjacent channels. Figure 2 shows the magnitude response of channels with a smooth change in frequency along the frequency axis.

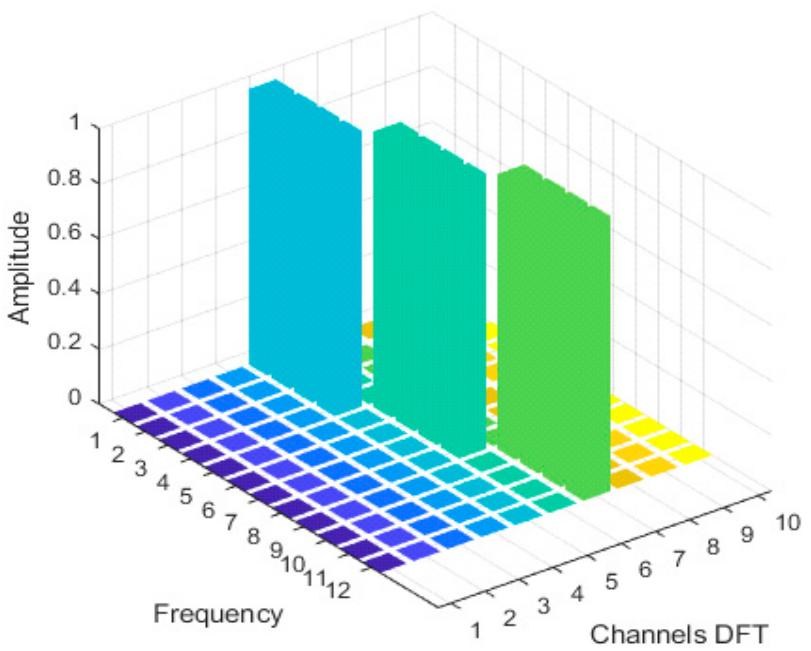

Figure 2. Frequency response of channels of the bandpass filters.

The three-dimensional representation of the spectrum makes it possible to estimate clearly the defects that are inherent to the discrete conversion of signals on a limited time interval.

We will use a mono-harmonic signal with a smooth frequency sweep to remove the frequency response. In the spectral area the mono-harmonic signal should show only one non-zero count in the channel with the number corresponding to the integer frequency value-the number of periods in the selected time interval. When the frequency changes, the spectral reference smoothly flows into the adjacent channel. The real frequency response of the DFT channels significantly differs from the ideal case, as is shown in Figure 3. 


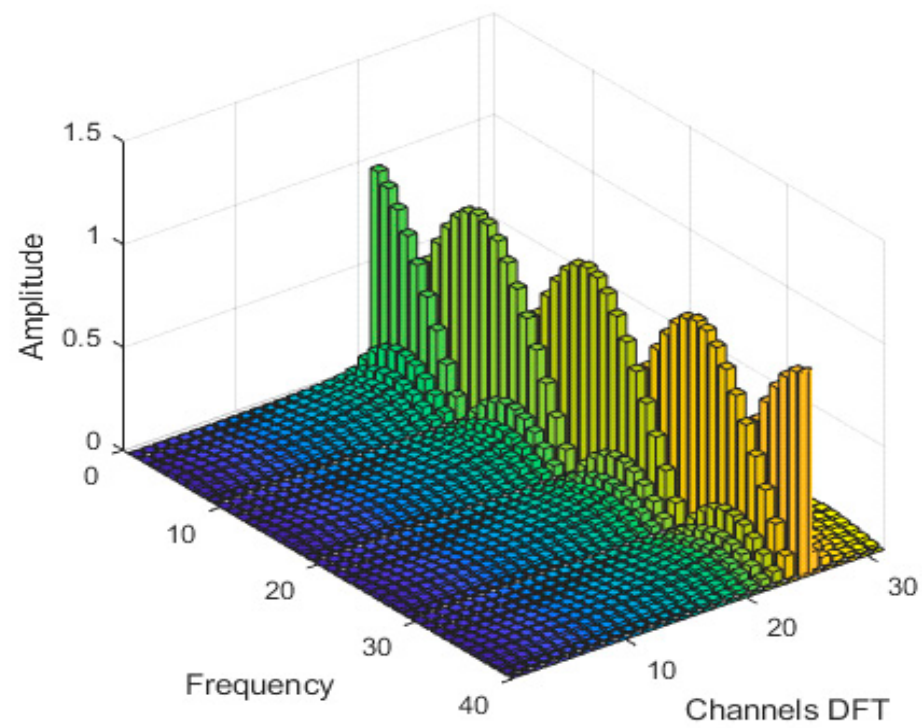

Figure 3. Frequency response of DFT channels.

Figure 3 allows visual assessing of the Fourier transform on a finite interval. The channel DFT axis refer to the spreading of the mono-harmonic signal spectrum along the adjacent DFT channels, which is caused by the presence of sidelobes of the frequency response of the channel. One can see their level and shape along the frequency axis. Non-zero transmission coefficients in a fairly wide frequency range lead to the penetration of frequencies into adjacent channels and degrade noise filtering.

To reduce the level of sidelobes the preliminary weighting of a signal sample by one of the numerous time windows, which smoothly descend at the edges of the range, is traditionally used [2].

The best of the window functions, such as Kaiser, Hanning, Dolph-Chebyshev, Kravchenko's atomic functions etc., provide suppression of the "tails" of the frequency response to levels $-80 \mathrm{~dB} \ldots$. $-100 \mathrm{~dB}$ [3]. However, the suppression of sidelobes is achieved by reducing the transform capability due to the expansion of the main lobe (Figure 4). This is a common drawback for all window functions: Whatever the shape of the weighing window is, it narrows the time interval of the signal, which is already short. Then the narrowed time interval inevitably leads to a broadening of the main lobe [4].

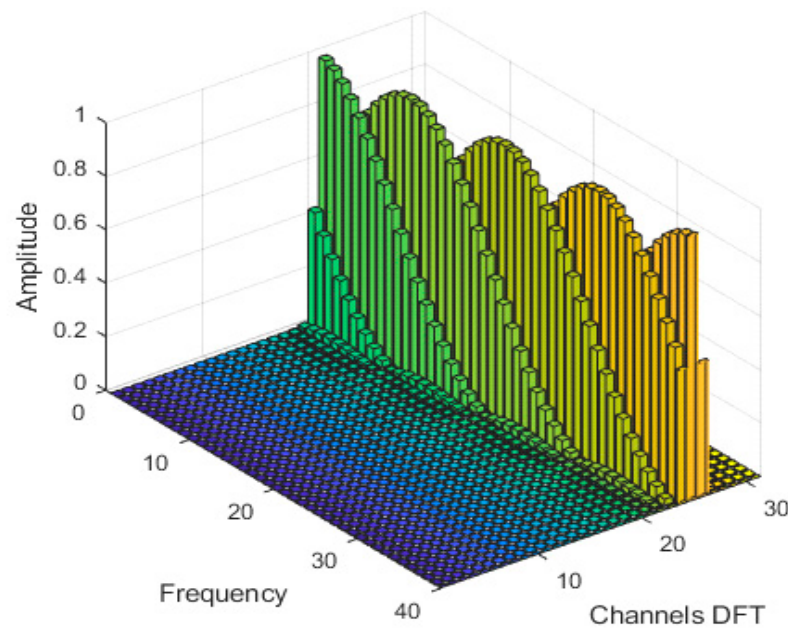

Figure 4. Frequency response of DFT channels using Hanning window weighting. 
There are many ways to increase the resolution of the DFT and remove sidelobes. The reference signal-cloning technique performs post-DFT correction of an accurate frequency shift [5]. The dual apodization method introduces a novel sidelobe and clutter suppression technique in ultrasound imaging called dual apodization with cross-correlation or DAX [6,7]. However, these methods have their own advantages and disadvantages. Their main drawback is a high computational complexity. Often, the complexity of resolution increase algorithms is many times greater than DFT itself, which prevents them from implementation in real-time systems.

In this paper, we propose a new method to reduce the sidelobes in the spectrum without increasing the width of the main lobe.

The rest of the paper is organized as follows. In Section 2, we introduce the idea of the proposed technique. In Section 3, the comparison between several window functions and the proposed method is shown, and frequency responses of the proposed method with different signal-to-noise ratio (SNR) are described. In Section 4, we present the examples of the proposed technique application. In Section 5, a brief discussion is given. In Section 6, some conclusions are made and the obtained simulation results are presented.

\section{Materials and Methods}

To analyze the signal in the frequency domain, the absolute value of complex samples obtained by the DFT procedure is not widely used. More often the instantaneous spectral density (SD) is calculated [8]:

$$
S_{x x}(f)=(\Delta t)^{2}\left|\sum_{n=-\infty}^{\infty} x_{n} e^{-2 \pi i f n \Delta t}\right|^{2},
$$

where $x_{n}$ is the signal samples, $\Delta t$ is the time interval of the analysis.

The operation of obtaining a SD is as follows:

$$
G(m)=S(m) \cdot S(m) *=(a(m)+j b(m))(a(m)-j b(m))=a(m) \cdot a(m)+b(m) \cdot b(m)
$$

where $S(m)$ is the spectral sample of the DFT, $S(m)^{*}$ is the complex-conjugate spectral sample of the DFT, $a(m)$ is the real part of the DFT sample, and $b(m)$ is the imaginary part of the DFT sample.

Squaring samples does not eliminate the sidelobes of the frequency response, but it is the closest analogue of the proposed method. The essential difference is that both adjacent parts of the same sample are subjected to multiplication:

$$
\mathrm{NC}=a(m) \cdot a(m+1)+b(m) \cdot b(m+1),
$$

where $a(m)$ is the material part of the DFT sample and $b(m)$ is the imaginary part of the DFT sample. We call this response neighbor spectrum sample components composition, or NC method [9].

The idea of this modification of the SD calculation is as follows. For any frequency value with any fractional part value (except of the rare case of integer value) the mono-harmonic signal falls into the overlap zone of two adjacent DFT channels (Figure 5), forming two adjacent spectral samples. Moreover, the phases in two channels are shifted by 180 degrees (Figure 6), regardless of the phase and frequency of the input signal. Equation (4) allows the selection of these components in an optimal way.

In Figure 5, the solid lines show the frequency response of two adjacent DFT channels, the dotted lines illustrate the frequency response of the proposed NC method. 


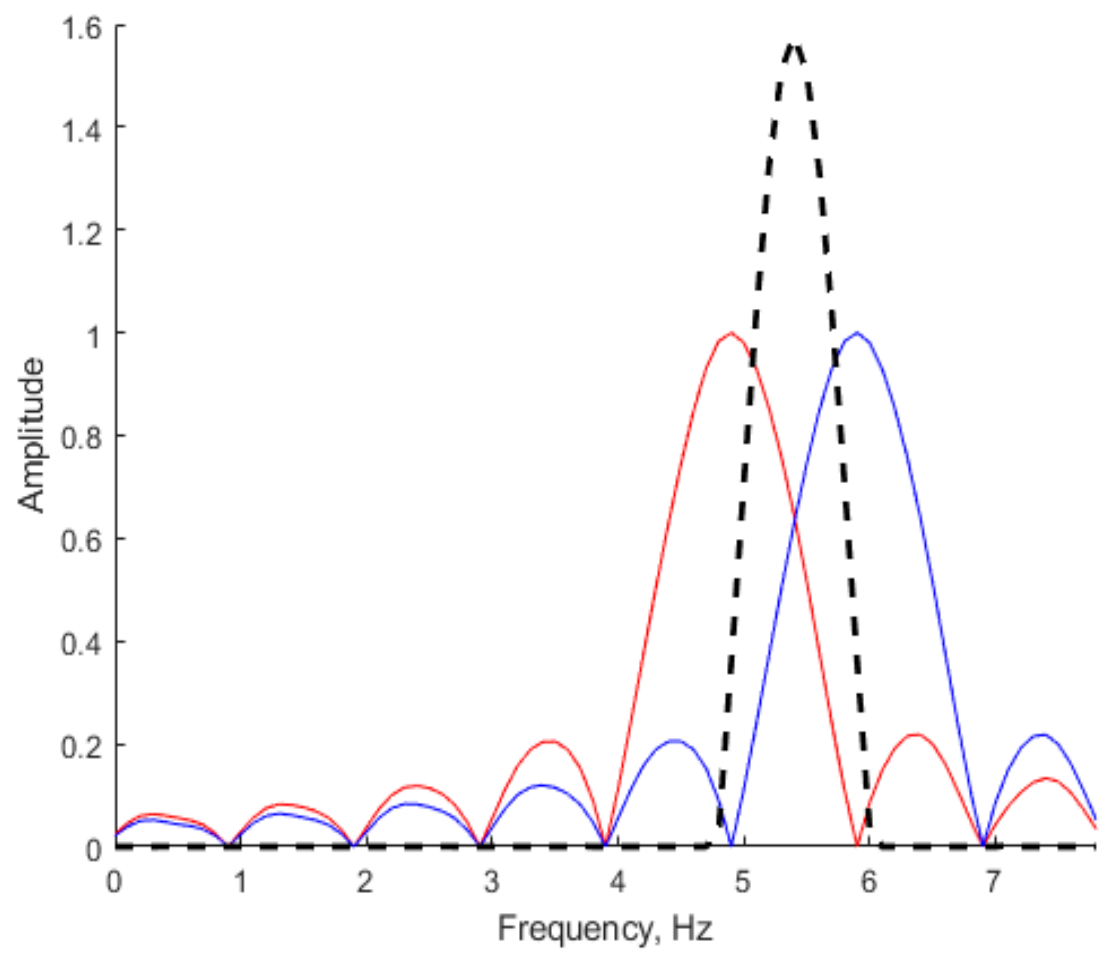

Figure 5. Magnitude response of the DFT and NC channels.

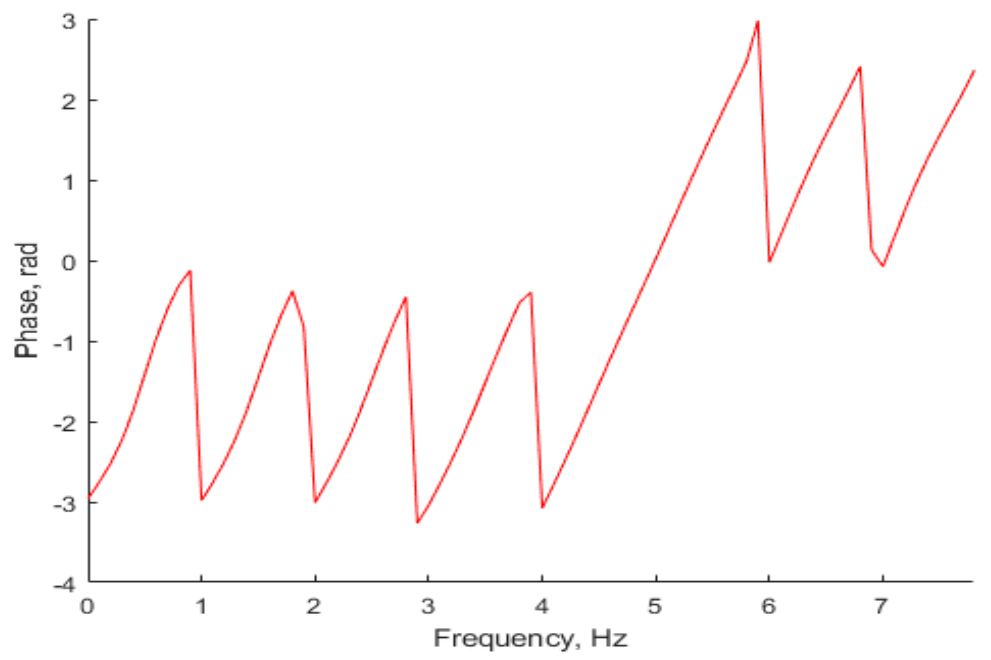

Figure 6. Phase-frequency characteristic of the DFT.

\section{Results}

The mutually opposite direction of the "split" spectral component of the signal leads to the fact that both terms in (4) have a negative value in the overlap zone of the main adjacent lobes and positive value for all sidelobes. The difference in the sign of the product allows for the zeroing of the small values of the spectrum lateral components (Figure 7), and at the same time change the sign of the remaining component. 


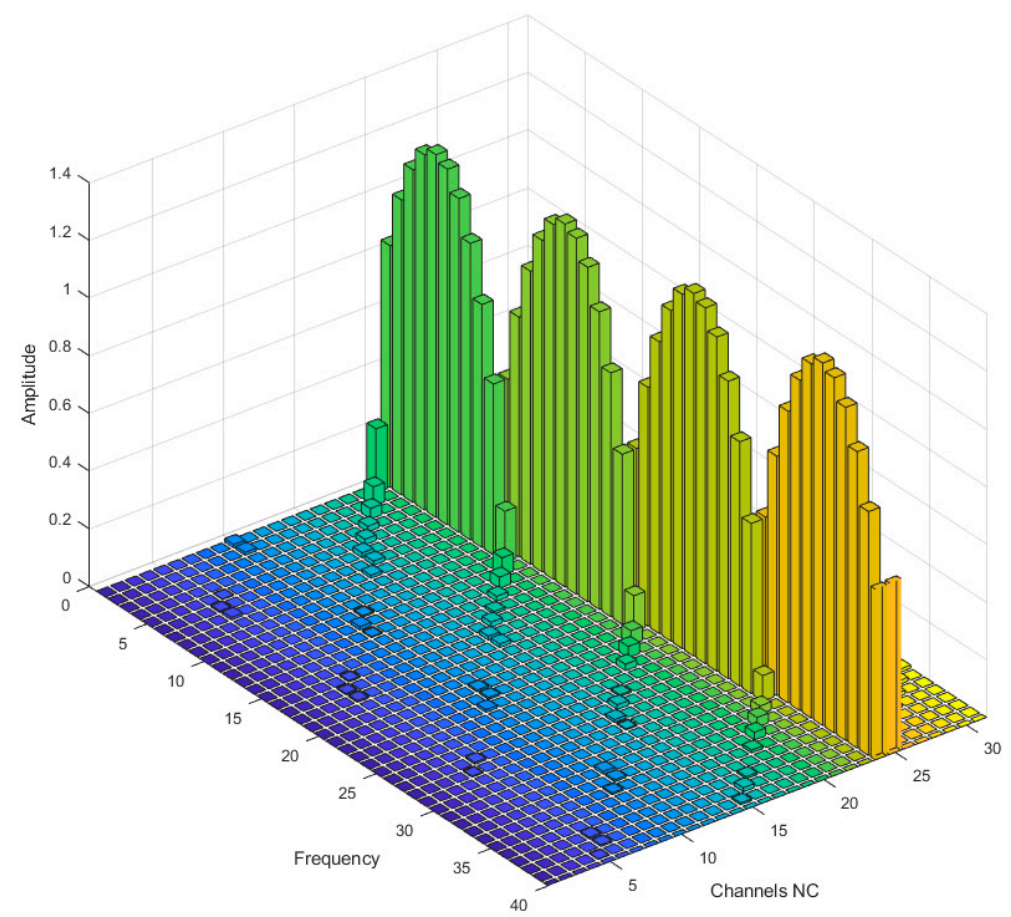

Figure 7. Frequency response of NC channels.

The following set of figures illustrate the amplitude spectra of the same noisy signal. Figure 8a in a logarithmic scale, Figure $8 \mathrm{~b}$ in a linear scale. White Gaussian noise with a signal-to-noise ratio $(\mathrm{SNR})=7 \mathrm{~dB}$ was added to the original signal.

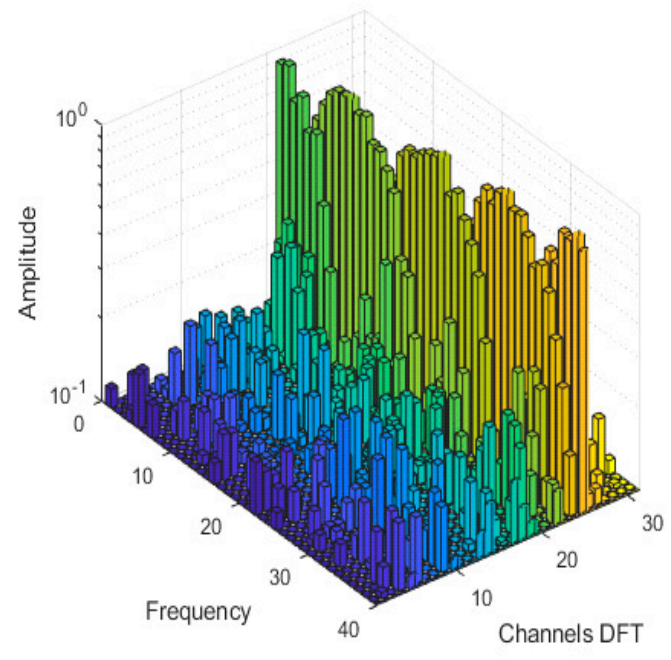

(a)

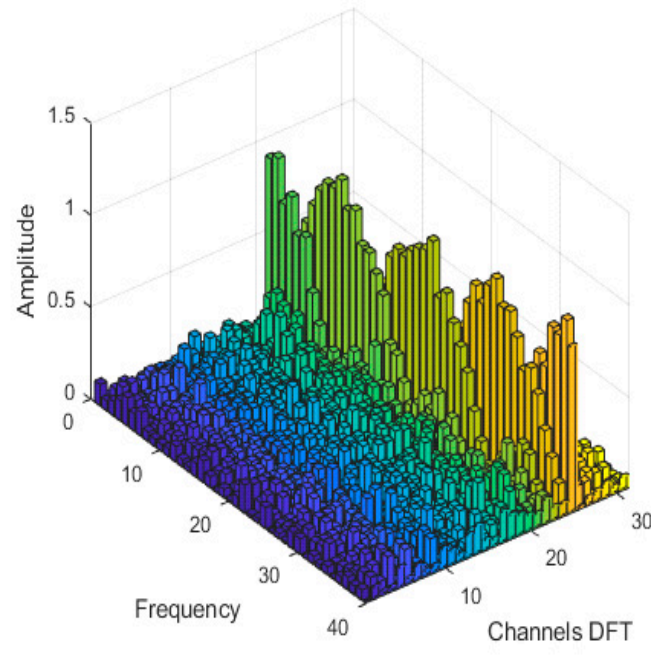

(b)

Figure 8. The magnitude response of the DFT channels: (a) Logarithmic scale; (b) linear scale.

The amplitude spectrum of the signal using the Hanning window transform is shown in Figure 9. 


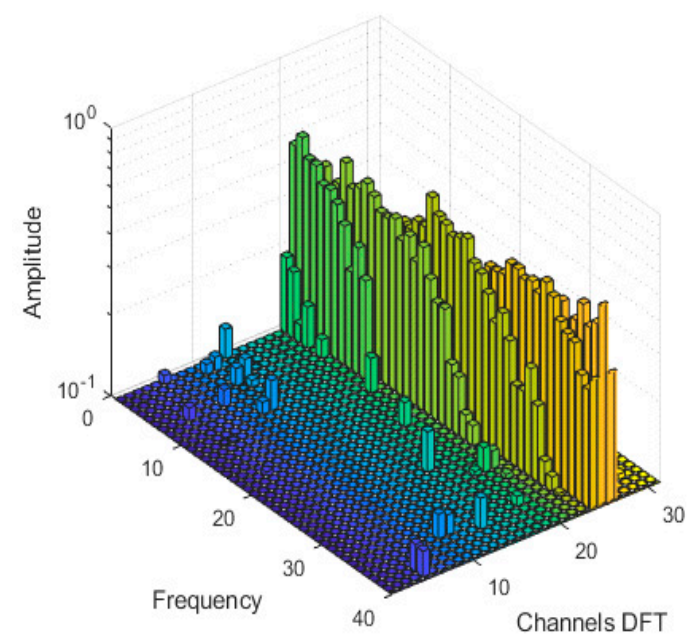

(a)

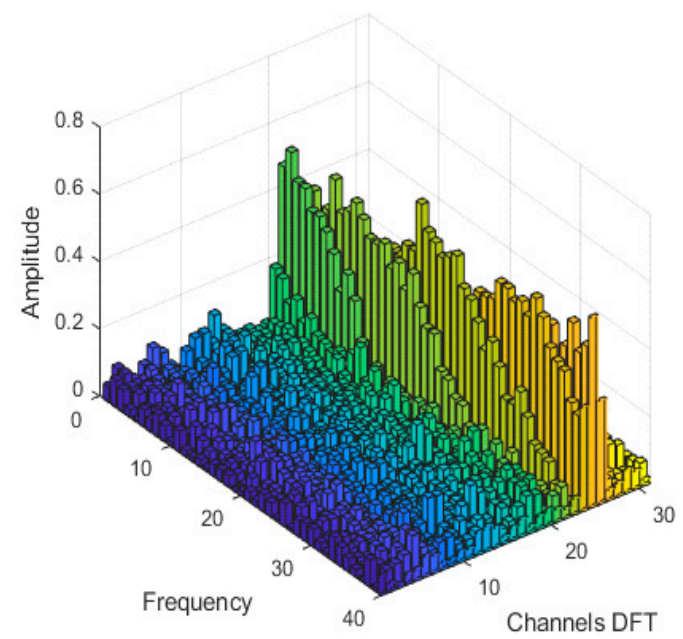

(b)

Figure 9. The magnitude response of the DFT channels using the Hanning window transform: (a) Logarithmic scale; (b) linear scale.

The amplitude spectrum of the signal transformed using the NC algorithm is shown in Figure 10.

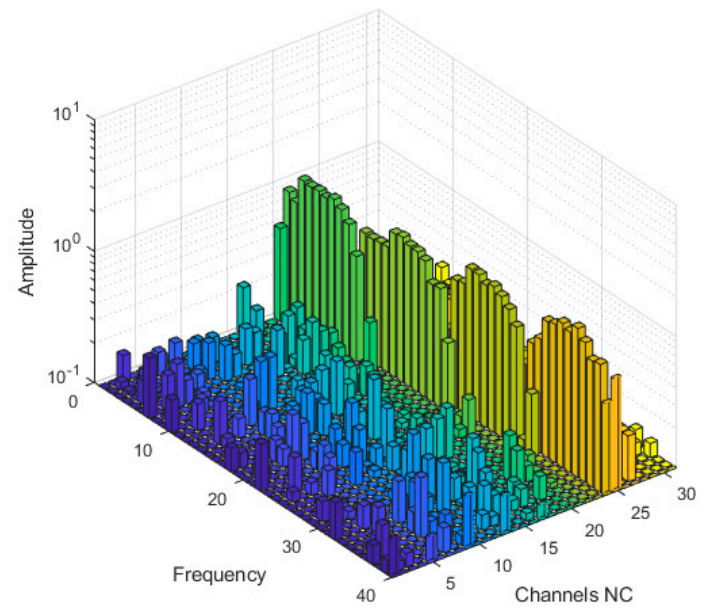

(a)

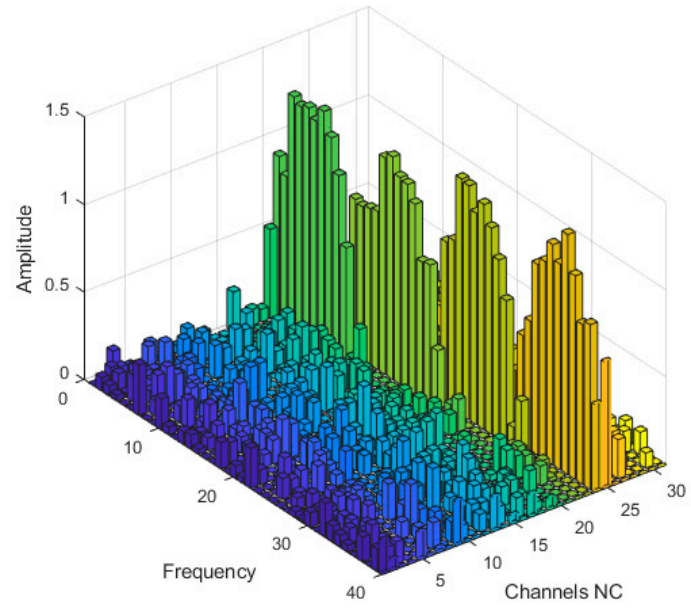

(b)

Figure 10. The magnitude response of NC channels: (a) Logarithmic scale; (b) linear scale.

Basic frequency responses of the window functions are given in Figure 11, allowing the comparison of the different windows with each other [10]:

- The normalized width of the frequency response main lobe at the level of $0.5(-3 \mathrm{~dB}) \Delta \mathrm{F}_{0.5}$.

- The normalized width of the main lobe of the frequency response at the zero level $\Delta \mathrm{F}_{0}$.

- Maximum sidelobe level $\gamma_{\max }$.

From the characteristics given in Table 1, one can see that the proposed NC method had the smallest width of the main lobe, in comparison with window functions, and was approximately 1.5 times smaller than in a case of the rectangular window. This minimizes spreading of the spectrum. Low levels of sidelobes means that most of them were close to zero, and the total weight of all sidelobes was $0.16 \%$ of the main lobe. The rectangularity of the main lobe (the ratio of the lobe width at the level of 0.5 to the width at the zeros level) was much higher than that of the approach based on windowing. 
A comparison of mono-harmonic signal detection using the DFT and NC techniques with different signal-to-noise ratios is presented in Figure 12.

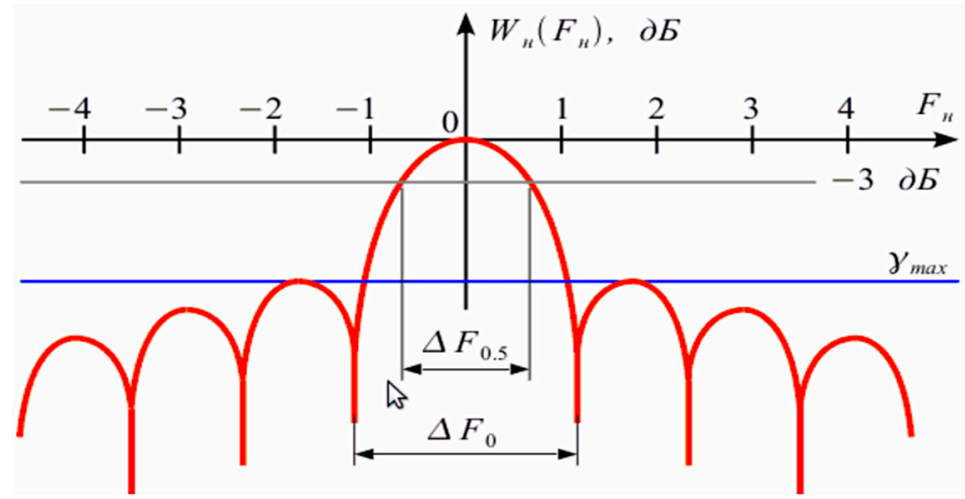

Figure 11. Characteristics of window functions.

Table 1. Properties of the main window functions and the proposed method [10].

\begin{tabular}{cccc}
\hline Window Functions & $\boldsymbol{\Delta} \boldsymbol{F}_{\mathbf{0}}$ & $\boldsymbol{\Delta} \boldsymbol{F}_{\mathbf{0 . 5}}$ & $\boldsymbol{\gamma}_{\text {max }} \mathrm{dB}$ \\
\hline Rectangle window & 2 & 0.89 & -13 \\
Sine window & 3 & 1.23 & -23 \\
Lanczos window, or sinc window & 3.24 & 1.3 & -26.4 \\
Bartlett window or triangular window & 4 & 1.33 & -26.5 \\
Hanning window & 4 & 1.5 & -31.5 \\
Bartlett-Hann window & 4 & 1.45 & -35.9 \\
Hamming window & 4 & 1.33 & -42 \\
Blackman window & 6 & 1.7 & -58 \\
Blackman-Harris window & 8 & 1.97 & -92 \\
Nuttall window & 8 & 1.98 & -93 \\
Blackman-Nuttall window & 8 & 1.94 & -98 \\
Flat top window & 10 & 3.86 & -69 \\
Gaussian window $\sigma=0.3$ & 8 & 1.82 & -65 \\
Gaussian window $\sigma=0.5$ & 3.4 & 1.2 & -31.5 \\
Gaussian window $\sigma=1.2$ & 2.2 & 0.94 & -15.5 \\
NC & 1.23 & 0.75 & -54 \\
\hline
\end{tabular}

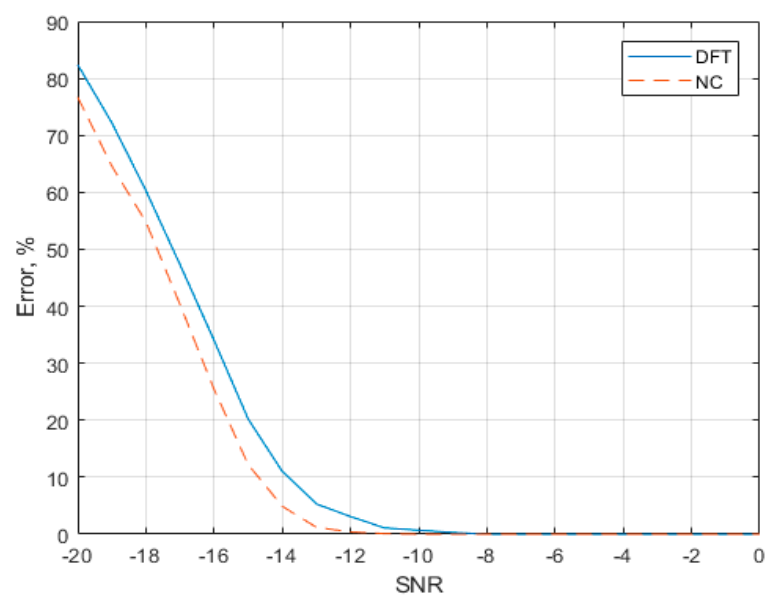

Figure 12. Comparison of mono-harmonic signal detection using DFT and NC.

For testing, we used a mono-harmonic signal with the fractional part equal to a quarter of the DFT sample increment width. A white Gaussian weighted noise was added to the signal. Then the amplitude spectrum and spectral density were calculated using NC. The maximum of the spectrum 
was estimated, and the frequency was measured at the maximum amplitude and then compared with the specified value. We performed 1000 experiments and calculated the error of the specified frequency detection.

The plot in Figure 12 shows that frequency detection using NC was, on average, 5\% more accurate than the standard DFT. In the range from lower -10 , the NC showed a zero error, unlike the DFT. Thus, the presented method was advantageous compared to the traditional DFT.

To increase the resolution of the frequency analysis, it was necessary to increase the length of the DFT, thus, if the resolution increases twice, the length of the signal analysis is also doubled, which results in the growth of the computational complexity [11]. It is known that the computational complexity of the DFT algorithm, particularly the FFT, is nlog (n). When we used the NC algorithm with the same resolution, some extra analysis was required to perform the operations. Figure 13 shows the growth of the computational complexity of the algorithms when the data length increased [12].

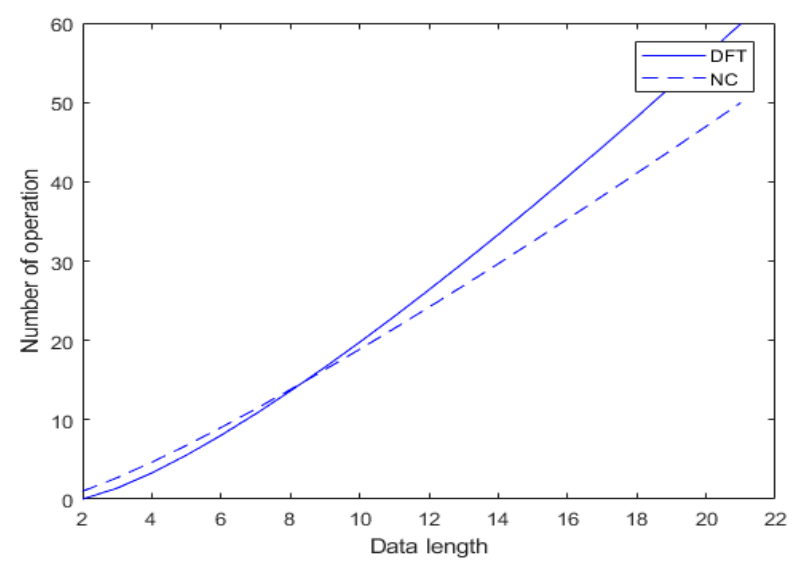

Figure 13. The growth of computational complexity with a change in the number of incoming samples.

One can see from Figure 13 that with an increase in the amount of data from nine samples or more, the number of FFT operations became larger compared to the NC. This shows that for reducing computational complexity, when calculating data of nine or more samples, it is more numerically expensive to use the $\mathrm{NC}$ algorithm.

Let us consider a mono-harmonic signal with a $20.3 \mathrm{~Hz}$ frequency. Since the frequency had a fractional part, discrete Fourier transform had leakage of the spectrum, see Figure 14.

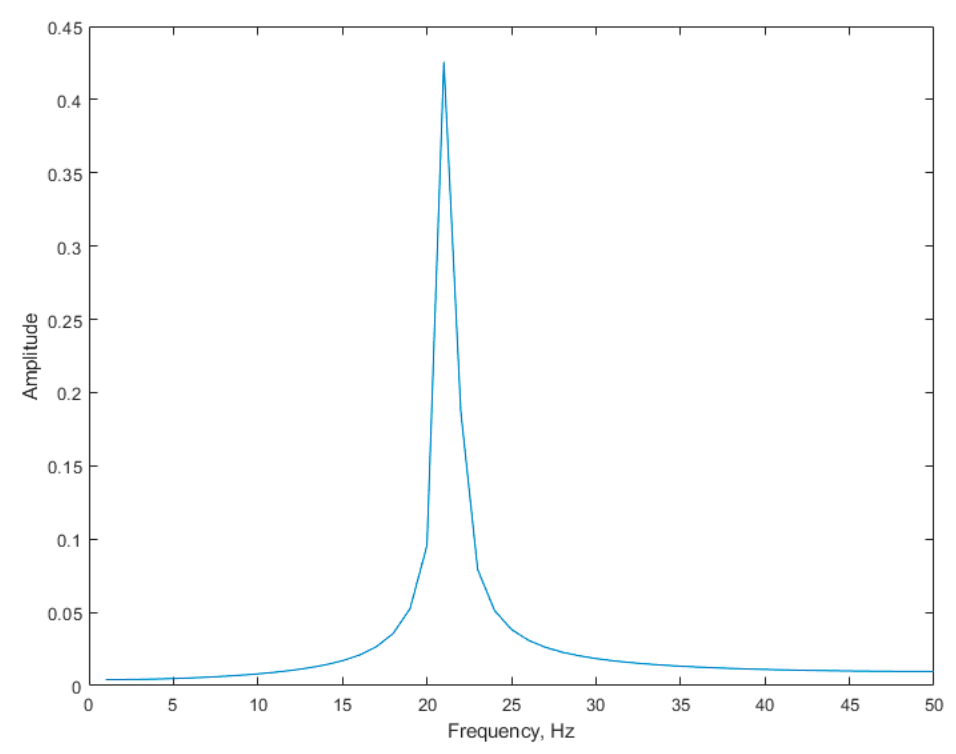

Figure 14. Spectrum of mono-harmonic signal with $20.3 \mathrm{~Hz}$. 
Figure 15 illustrates the results obtained by the NC algorithm. One can see that the algorithm eliminated leakage of the spectrum, since it did not have sidelobes, and; therefore, only one sample was present. The operation of the algorithm was considered in more detail in following examples.

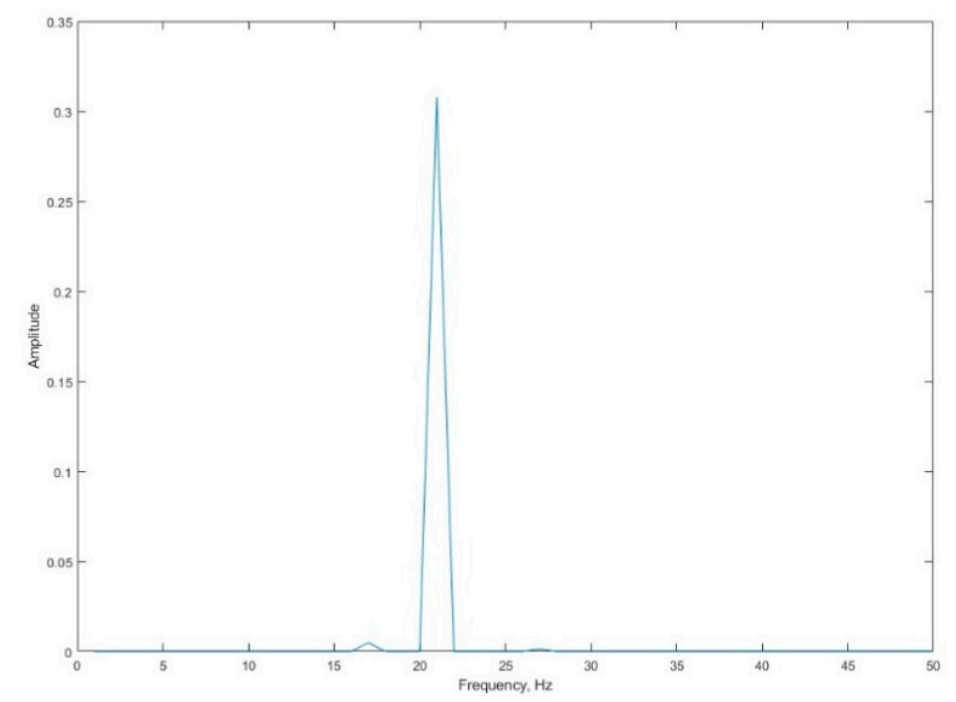

Figure 15. NC spectrum of mono-harmonic signal with $20.3 \mathrm{~Hz}$.

To show the increase of the frequency resolution when using the proposed technique, we considered two examples. Figure 16 shows the first example. Here we see the spreading of the Fourier spectrum of a mono-harmonic signal with a frequency of $20.5 \mathrm{~Hz}$, and the spectrum when using the proposed technique.
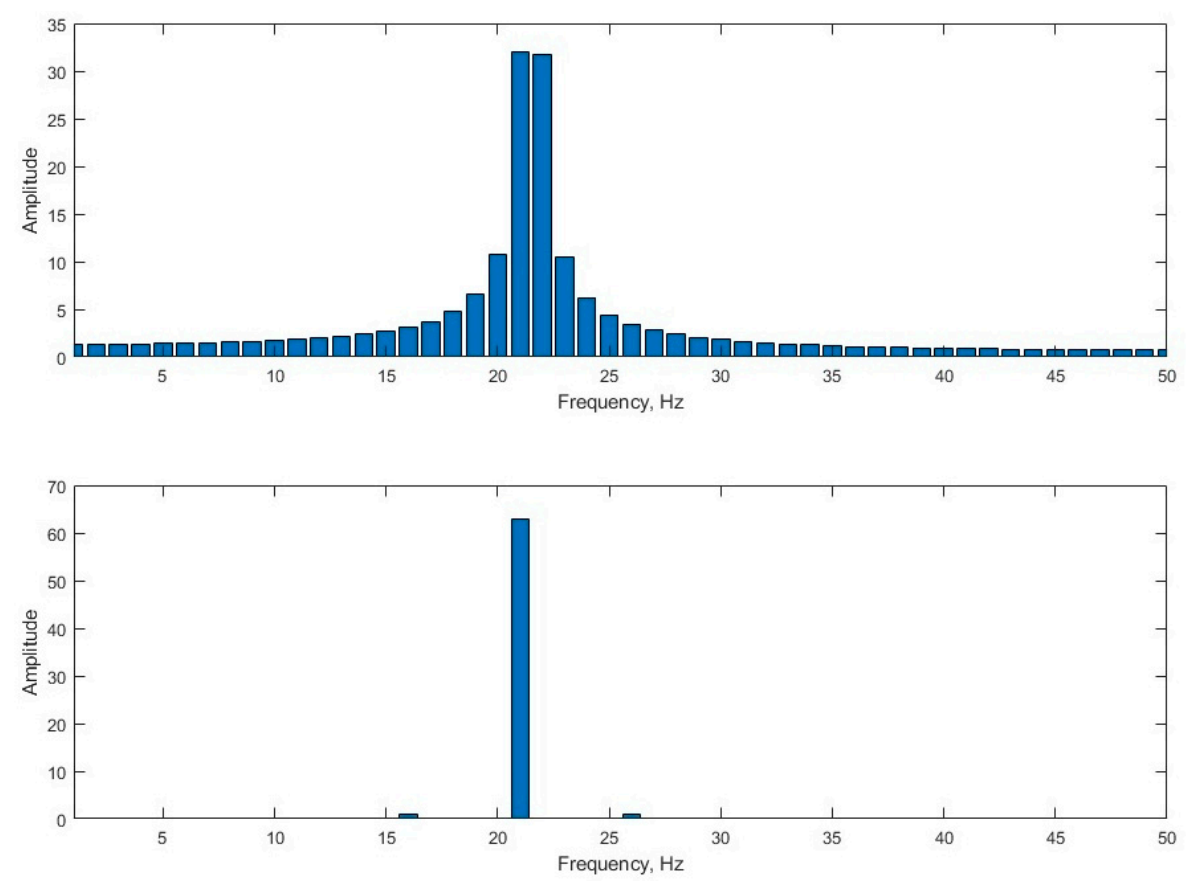

Figure 16. DFT (up) and NC (down) spectrum of mono-harmonic signal with $20.5 \mathrm{~Hz}$.

Figure 17 shows the spectrum of two mono-harmonic signals, with frequencies of 19.8 and $20.2 \mathrm{~Hz}$, using the DFT, and the spectrum of the same signals using the proposed technique. 

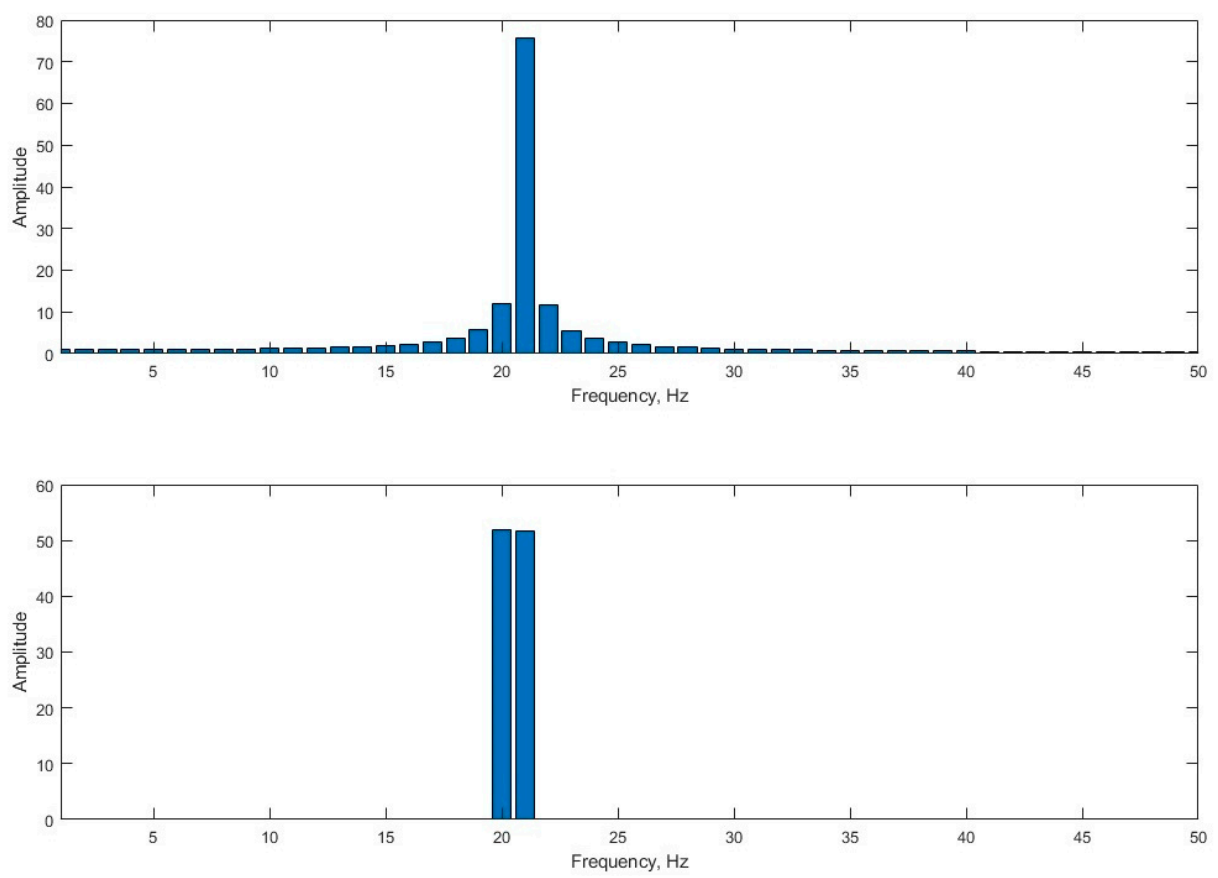

Figure 17. DFT (up) and NC (down) spectrum of two mono-harmonic signals, with 19.8 and $20.2 \mathrm{~Hz}$.

\section{Examples}

Most recent methods for vibration diagnostics are based on the vibration analysis of industrial machines and equipment. These methods form the basis of functional diagnostics, meanwhile the operation modes can be very different: From steady (nominal or special) to transient, including starting, impulse, etc. During the functional diagnostics of machines and equipment by vibration, the information contained in the characteristics of the oscillatory forces and the properties of the oscillatory system is usually discovered [13]. Thus, before the vibrational analysis begins, there is no sufficiently accurate information about the oscillatory forces or the oscillatory system [14].

We can suppose that more complex methods of vibration analysis would give better results.

Practically, in rotary machines, there are no impact components among the low-frequency vibrational forces, and random components make a very small contribution. Therefore, only a small number of resonances have transfer characteristics. Thus, one can accurately determine characteristics and vibrational forces of an oscillating system by low-frequency vibration (usually up to frequencies of about $1000 \mathrm{~Hz}$ ) of a rotary-type machine. The spectral analysis of vibrations measured at different points and directions becomes the main method for obtaining diagnostic information. Spectral analysis of low-frequency machine vibrations is one of the main fields of functional diagnostics, which allow for the detection of up to half of possible defects of rotary-type machines long before an emergency case occurs.

Piezoelectric sensors are common hardware for abovementioned tasks. Usually these sensors have an internal DSP processor for calculating FFT. However, this Fourier transform has limitations, since it is hardware-implemented and works over a limited period; therefore, the question of increasing the FFT resolution arises. Figure 18 shows the amplitude spectrum of a faulty asynchronous motor in steady state. The measurements were carried out on the engine Elnor $1.1 \mathrm{~kW}$ [15]. 


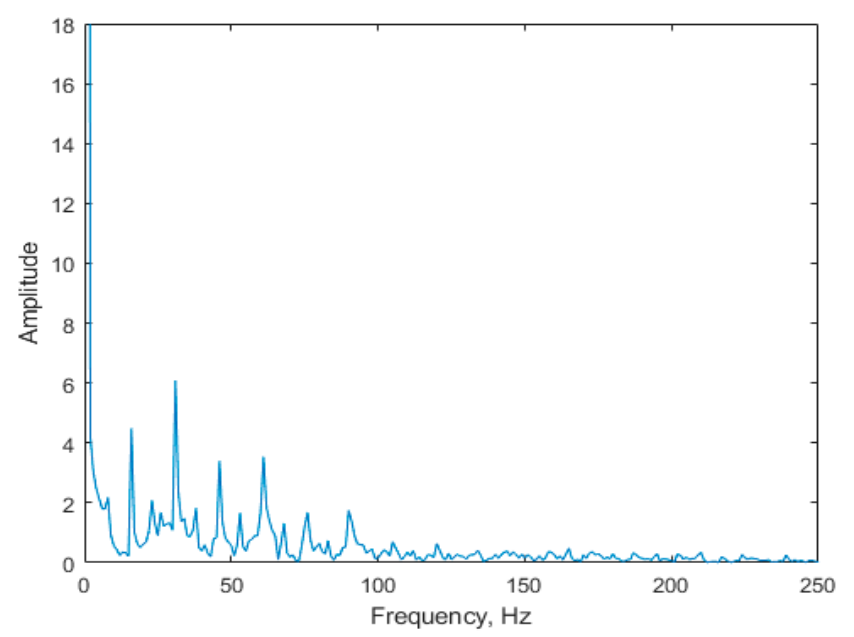

Figure 18. Amplitude spectrum of a faulty asynchronous motor in steady state.

The engine have an additional mono-harmonic vibrations, which indicate the wear of internal parts. Monitoring the frequency response of the engine can predict the location of possible failure and identify the problem on early stage. To perform this, we determined the oscillation frequency with the desired accuracy. However, it may happen that the central frequency of oscillation falls between the main lobes of the FFT. This situation is illustrated in Figure 19.

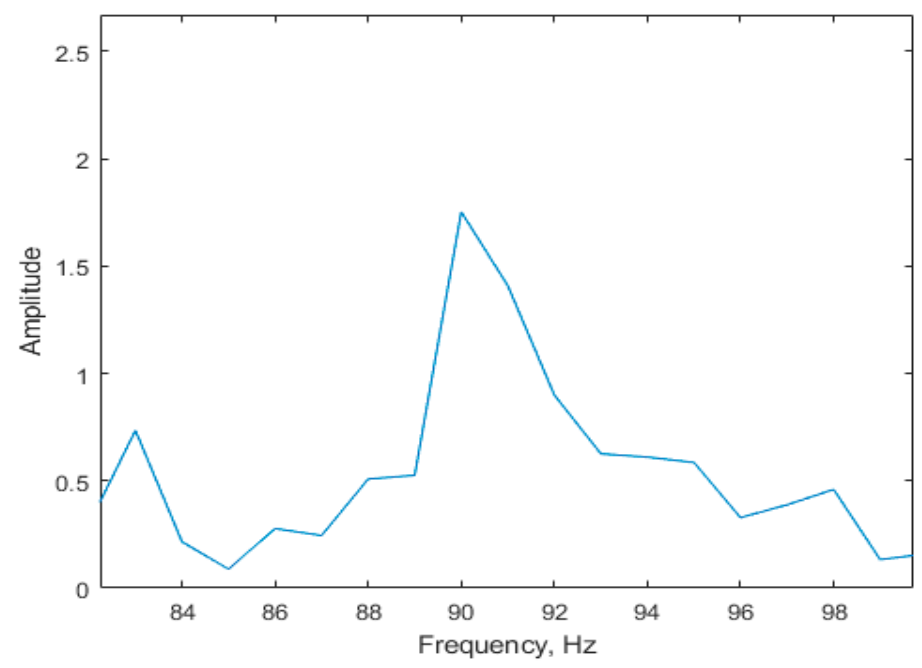

Figure 19. Amplitude spectrum of a faulty asynchronous motor in steady state.

Using the proposed NC technique, one can increase the resolution of the FFT and specify the oscillation frequency.

The peak in Figure 20 indicates that the oscillation frequency is exactly between the DFT channels, thus the center frequency of the oscillation is approximately $90.5 \mathrm{~Hz}$. If these were two different modes, NC method would distinguish it as well. 


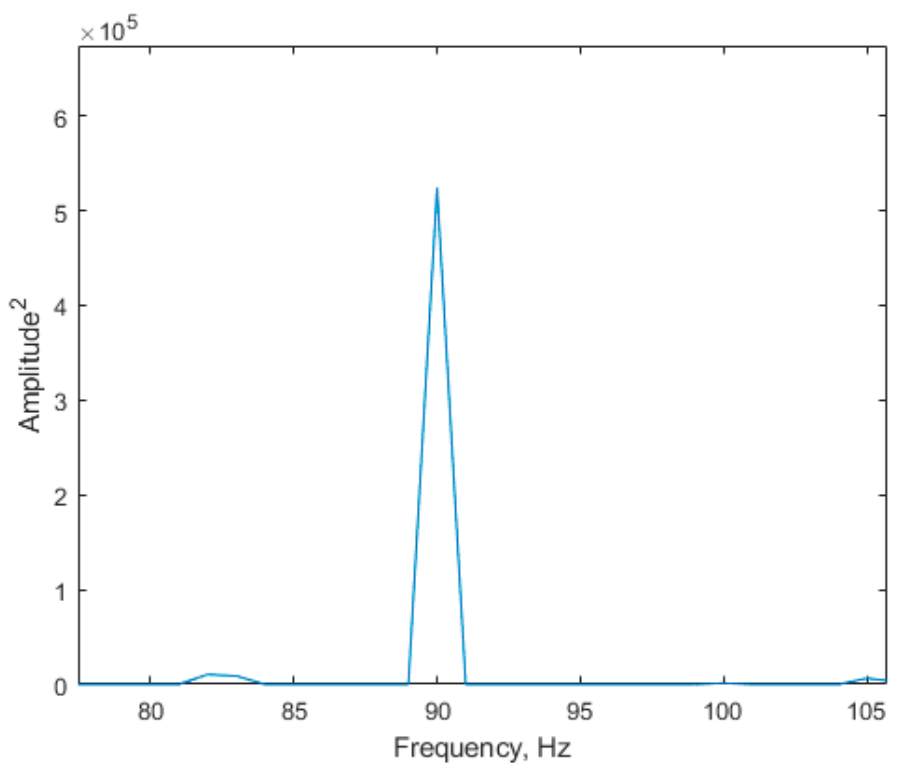

Figure 20. Amplitude spectrum of a faulty asynchronous motor in steady state built with NC technique.

Our method allows one to double the resolution of the DFT, clarifying the fractional parts of the DFT grid.

At present, advanced driver assistance system (ADAS) systems are being actively developed, which include short-range radars. Short-range radars (SRR) are radar systems that circle a car while driving to detect possible obstacles, such as other cars, walls, pedestrians, etc. These systems also should automatically trigger the safety measures, such as pre-tension of seat belts and air bags activation. This also may include automatic braking to avoid a collision [16].

A simplified diagram of the short-range radar is presented in Figure 21 [16].

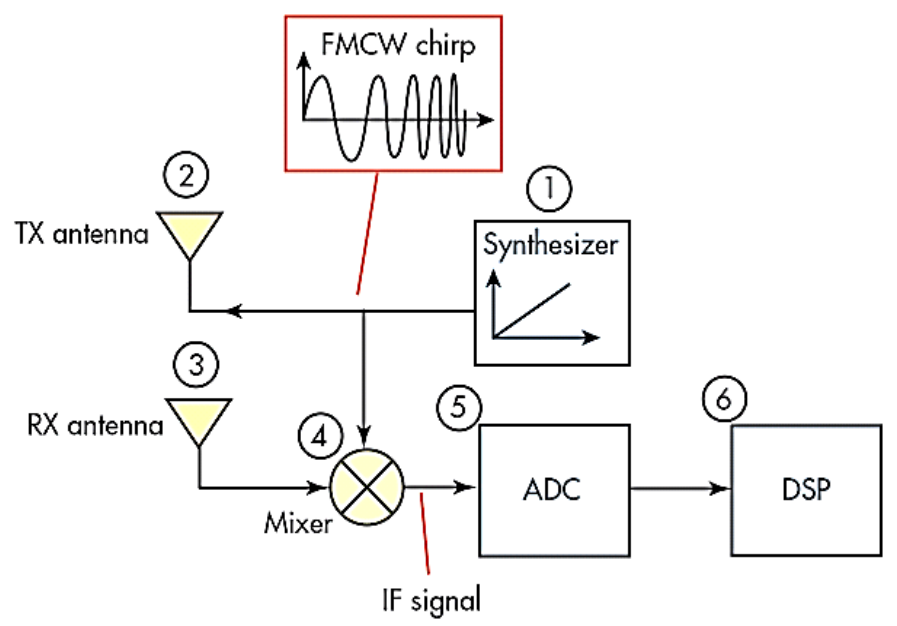

Figure 21. Chirp short-range radar scheme.

In the signal generator (Figure 21, (1)), the chirp signal is synthesized and fed to the emitting antenna (Figure 21, (2)), and the same signal is transmitted to the multiplier block (Figure 21, (4)), which multiplies the chirp signal on the received signal from the antenna (Figure 21, (3)). After multiplication, the signal is transmitted to the analog-to-digital converter (ADC) (Figure 21, (5)) and the subsequent information is processed. After the ADC, data is transferred to the fast Fourier transform block. Since the transmitter power is rather low, the signal-to-noise ratio of the received signals is quite small. The amplitude spectrum from a record of one set of points with an ADC is shown in Figure 22. Measurements were made with the Infineon $24 \mathrm{GHz}$ radar kit [17]. 


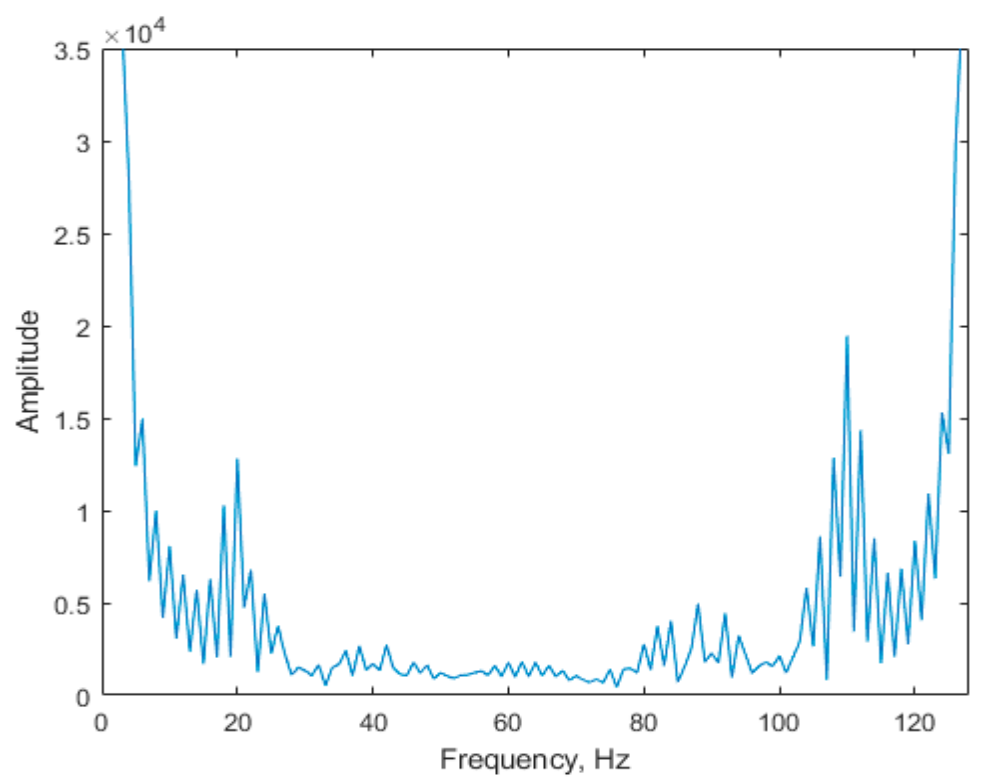

Figure 22. Amplitude spectrum of a received signal.

One can see from obtained data that there were two objects in front of the radar that could be detected at frequencies of 20 and $110 \mathrm{~Hz}$. There also were a lot of spurious frequencies, and visible spreading of the spectrum can be observed. Applying the NC algorithm on the same data, we got the following amplitude spectrum (Figure 23).

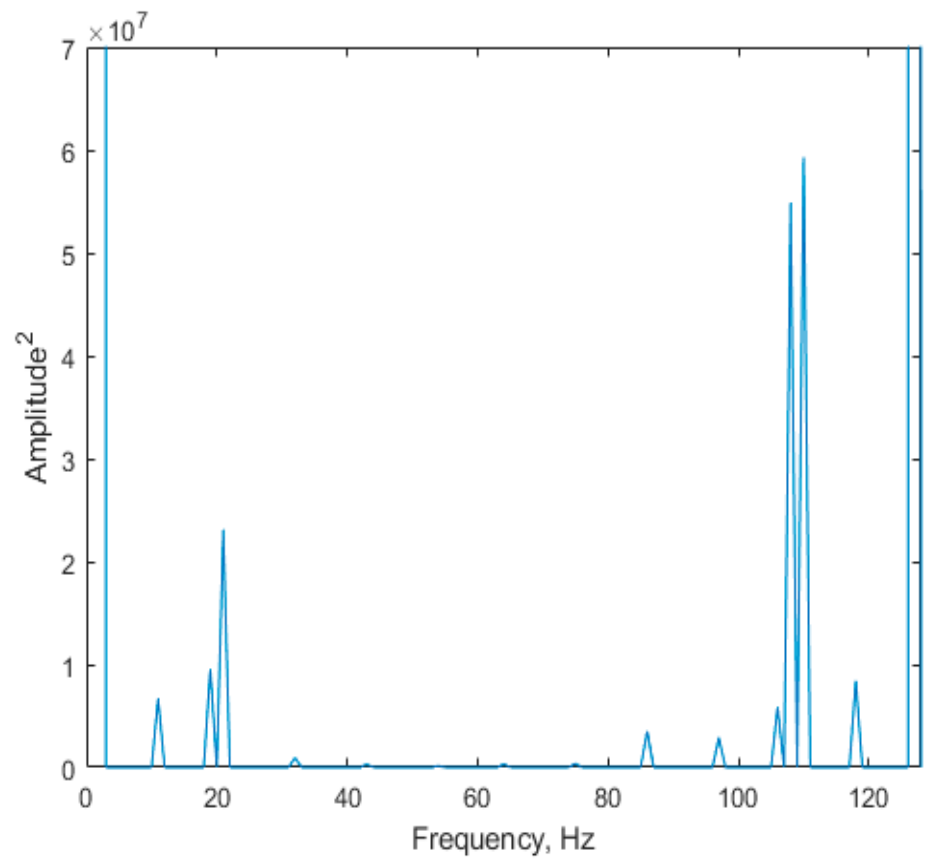

Figure 23. Amplitude NC spectrum of a received signal.

The signals reflected from the studied objects can be more clearly distinguished from the spectrum, and the effect of spectrum spreading also disappeared.

\section{Discussion}

The proposed NC method can be applied in various fields of technology where it is required to perform spectral analysis of signals. For example, a good task to use the proposed NC method is 
described in [18]. Comparing with other methods that use phase information to estimate precisely the frequency of a mono-harmonic signal [19], we can conclude that most of them estimate the frequency without forming channels. This makes such methods applicable for specification of the frequency, but less intended to obtain an amplitude spectrum. Our method has advantages over existing methods, such as the window Fourier transform, namely, the more rectangular shape of the main lobe and the lower level of sidelobes. A small number of operations to calculate the NC transform allows for the achievement of better results without a notable increase of the computational complexity. In our further research, we plan to investigate this approach considering the influence of various effects on the accuracy of the analyzed data.

\section{Conclusions}

To conclude the paper, we listed the main advantages of the proposed method:

- The width of the main lobe is the smallest among all known window functions, 1.5 times smaller than for the rectangular window. This minimizes spectrum spreading accordingly.

- Low level of sidelobes, most of which are zeros; the total weight of all sidelobes is $0.16 \%$ of the main lobe.

- The rectangularity of the main lobe, calculated as the ratio of the width of the lobe at 0.5 to the width at the level of zeros, is much higher than that of any existing windows.

- The location of the main lobe maximum corresponds to the middle of the interval between spectral readings, which is most convenient for the combined DFT-NC processing, which guarantees a two-fold increase in resolution.

Author Contributions: Conceptualization, I.K.; data curation, D.K., D.B., and A.S.; formal analysis, I.K., D.K., and A.S.; investigation, D.K. and V.G.; methodology, I.K.; project administration, I.K. and D.K.; resources, D.B. and A.S.; software, V.G.; validation, D.B. and V.G.; visualization, V.G. and A.S.; writing—original draft, I.K., D.K., D.B., and V.G.; writing-review and editing, D.B. and A.S.

Funding: This research received no external funding.

Conflicts of Interest: The authors declare no conflict of interest.

\section{References}

1. Brigham, E.O. The Fast Fourier Transform and Its Applications; Prentice Hall: Englewood Cliffs, NJ, USA, 1998.

2. Rabiner, L.R.; Gold, B. Theory and Application of Digital Signal Processing; Prentice Hall: Englewood Cliffs, NJ, USA, 1975.

3. Hansen, R.C. Phased Array Antennas; John Wiley \& Sons: New York, NY, USA, 2009.

4. Oppenheim, A.; Schafer, R. Discrete-Time Signal Processing; Prentice Hall: Upper Saddle River, NJ, USA, 1999.

5. Priotti, P. Apparatus, and Associated Method, for Effectuating Post-FFT Correction of Fine Frequency Offset. U.S. Patent US20040120410A1, 20 December 2002.

6. Guenther, D.A.; Walker, W.F. Optimal Apodization Design for Medical Ultrasound Using Constrained Least Squares Part II: Simulation Results. IEEE Trans. Ultrason. Ferroelectr. Freq. Control 2007, 54, 343-358. [CrossRef]

7. Seo, C.H.; Yen, J.T. Sidelobe Suppression in Ultrasound Imaging using Dual Apodization with Cross-correlation. IEEE Trans. Ultrason. Ferroelectr. Freq. Control 2008, 55, 2198-2210. [PubMed]

8. Norton, M.P.; Karczub, D.G. Fundamentals of Noise and Vibration Analysis for Engineers; Cambridge University Press: Cambridge, UK, 2003.

9. Kanatov, I.I.; Gul'vansky, V.V.; Kaplun, D.I. Method of Decrease of Discrete Fourier Transform Sidelobes without Window Functions. In Proceedings of the 7th Mediterranean Conference on Embedded Computing (MECO), Budva, Montenegro, 10-14 June 2018; pp. 1-4.

10. Spectral Analysis over a Limited Time Interval. Window Functions. Available online: http://www.dsplib. $\mathrm{ru} /$ content/win/win.html (accessed on 14 August 2018). 
11. Lin, H.C. Separation of adjacent interharmonics using maximum energy retrieving algorithm. IET Sci. Meas. Technol. 2016, 10, 92-99. [CrossRef]

12. Lin, H.; Ye, Y.-C.; Huang, B.-J.; Su, J.-L. Bearing vibration detection and analysis using enhanced fast Fourier transform algorithm. Adv. Mech. Eng. 2016, 8, 80. [CrossRef]

13. Shearman, A.R.; Solovyov, A.B. Practical Vibration Diagnostics and Monitoring of Mechanical Equipment; Shearman, A.: Moscow, Russia, 1996.

14. Milan, S.K.; Sumathi, P. Vibration parameter estimation methods for ultrasonic measurement systems-A review. IET Sci. Meas. Technol. 2015, 9, 492-504.

15. Motors. Available online: https://www.elnor.be/browse/products/ (accessed on 14 August 2018).

16. Infineon Technologies. Available online: https://www.infineon.com/cms/en/product/sensor/radarsensor-ics/24ghz-radar/ (accessed on 22 July 2018).

17. AWR1642. Available online: https://www.ti.com/product/AWR1642/technicaldocuments (accessed on 22 July 2018).

18. Lv, Y.; Wu, Y.; Wang, H.; Qiu, L.; Jiang, J.; Sun, Y. An Inverse Synthetic Aperture Ladar Imaging Algorithm of Maneuvering Target Based on Integral Cubic Phase Function-Fractional Fourier Transform. Electronics 2018, 7, 148. [CrossRef]

19. Engelberg, S.; Chalom, E. Measuring the Spectral Content of a Signal: An Introduction. IEEE Inst. Meas. Mag. 2010, 13, 34-38. [CrossRef]

(C) 2019 by the authors. Licensee MDPI, Basel, Switzerland. This article is an open access article distributed under the terms and conditions of the Creative Commons Attribution (CC BY) license (http://creativecommons.org/licenses/by/4.0/). 